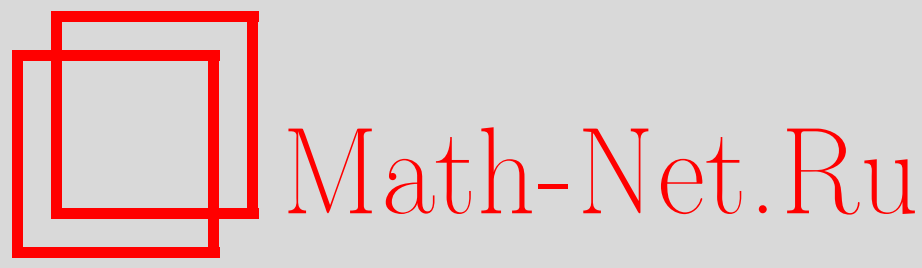

М. М. Рахматуллаев, О слабо периодических мерах Гиббса модели Изинга с внешним полем на дереве Кэли, TMФ, 2015, том 183, номер 3, 434-440

DOI: https://doi.org/10.4213/tmf8796

Использование Общероссийского математического портала Math-Net.Ru подразумевает, что вы прочитали и согласны с пользовательским соглашением http://www.mathnet.ru/rus/agreement

Параметры загрузки:

IP: 54.198 .67 .100

26 апреля 2023 г., $17: 16: 14$

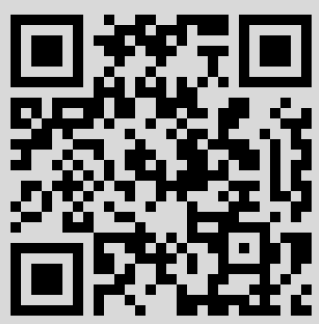




\section{О СЛАБО ПЕРИОДИЧЕСКИХ МЕРАХ ГИББСА МОДЕЛИ ИЗИНГА С ВНЕШНИМ ПОЛЕМ НА ДЕРЕВЕ КЭЛИ}

На дереве Кэли изучается слабо периодическая мера Гиббса для модели Изинга с внешним полем. Для антиферромагнитной модели Изинга с внешним полем при некоторых условиях на параметры доказано существование не менее чем двух слабо периодических мер Гиббса.

Ключевые слова: дерево Кэли, мера Гиббса, модель Изинга с внешним полем, слабо периодические меры.

DOI: $10.4213 / \operatorname{tmf} 8796$

\section{1. ВВЕДЕНИЕ}

Одна из основных проблем при изучении гамильтониана - это описание всех отвечающих ему предельных мер Гиббса. Для модели Изинга известно, что множество таких мер образует непустое, выпуклое, компактное подмножество в множестве всех вероятностных мер. Задача полного описания элементов этого множества далека от своего завершения. Для модели Изинга с нулевым внешним полем описаны трансляционно-инвариантные (см., например, монографии [1]-[4]), периодические [1], [5] и континуальные множества непериодических [1], [5] мер Гиббса для модели Изинга на дереве Кэли. С анализом трансляционно-инвариантных и периодических мер Гиббса для модели Изинга с внешним полем можно ознакомиться в работах [1], [2], [6], [7].

С целью получения более широкого множества мер Гиббса в работах [8]-[11] введены более общие понятия периодической меры Гиббса, а именно слабо периодические меры Гиббса, и доказано существование таких мер для модели Изинга на дереве Кэли. В работах [8] и [9] на некоторых инвариантных множествах при некоторых условиях на параметры найдены слабо периодические (непериодические) меры Гиббса для модели Изинга на дереве Кэли. Но для модели Изинга с внешним полем слабо периодическая мера Гиббса до сих пор не изучалась.

${ }^{*}$ Институт математики при Национальном университете Узбекистана, Ташкент, Узбекистан. E-mail: mrahmatullaev@rambler.ru 
В настоящей работе рассмотрена модель Изинга с внешним полем и при некоторых условиях на параметры доказано существование слабо периодических (непериодических) мер Гиббса. Структура работы такова: в разделе 2 даются необходимые определения и постановка задачи, раздел 3 посвящен изучению слабо периодических мер Гиббса, соответствующих нормальным делителям индекса два.

\section{2. ОПРЕДЕЛЕНИЯ И ПОСТАНОВКА ЗАДАЧИ}

Пусть $\tau^{k}=(V, L), k \geqslant 1$, есть дерево Кэли порядка $k$, т. е. бесконечное дерево, из каждой вершины которого выходит равно $k+1$ ребер; здесь $V$ - множество вершин, $L$ - множество ребер дерева $\tau^{k}$. Известно, что $\tau^{k}$ можно представить как $G_{k}-$ свободное произведение $k+1$ циклических групп второго порядка. Для произвольной точки $x^{0} \in V$ положим

$$
W_{n}=\left\{x \in V \mid d\left(x^{0}, x\right)=n\right\}, \quad V_{n}=\bigcup_{m=0}^{n} W_{m}, \quad L_{n}=\left\{\langle x, y\rangle \in L \mid x, y \in V_{n}\right\},
$$

где $d(x, y)$ - расстояние между вершинами $x$ и $y$ на дереве Кэли, т. е. число ребер кратчайшего пути, соединяющего вершины $x$ и $y$.

Пусть $\Phi=\{-1,1\}$ и $\sigma \in \Omega=\Phi^{V}-$ конфигурация, т. е.

$$
\sigma=\{\sigma(x) \in \Phi, x \in V\} .
$$

Пусть $A \subset V$. Обозначим через $\Omega_{A}$ пространство конфигураций, определенных на множестве $A$ и принимающих значения из $\Phi=\{-1,1\}$.

Рассмотрим гамильтониан модели Изинга с внешним полем

$$
H(\sigma)=-J \sum_{\langle x, y\rangle \in L} \sigma(x) \sigma(y)-\lambda \sum_{x \in V} \sigma(x),
$$

где $J, \lambda \in \mathbb{R}$ и $\langle x, y\rangle$ - ближайшие соседи.

Пусть $h_{x} \in \mathbb{R}, x \in V$. Для каждого $n$ определим меру $\mu_{n}$ на $\Omega_{V_{n}}$, полагая

$$
\mu_{n}\left(\sigma_{n}\right)=Z_{n}^{-1} \exp \left\{-\beta H\left(\sigma_{n}\right)+\sum_{x \in W_{n}} h_{x} \sigma(x)\right\},
$$

где $\beta=1 / T$ ( $T$ - температура, $T>0), \sigma_{n}=\left\{\sigma(x), x \in V_{n}\right\} \in \Omega_{V_{n}}, Z_{n}^{-1}$ - нормировочный множитель и

$$
H\left(\sigma_{n}\right)=-J \sum_{\langle x, y\rangle \in L_{n}} \sigma(x) \sigma(y)-\lambda \sum_{x \in V_{n}} \sigma(x) .
$$

Условие согласованности для мер $\mu_{n}\left(\sigma_{n}\right), n \geqslant 1$, задается равенством

$$
\sum_{\sigma^{(n)}} \mu_{n}\left(\sigma_{n-1}, \sigma^{(n)}\right)=\mu_{n-1}\left(\sigma_{n-1}\right),
$$

где $\sigma^{(n)}=\left\{\sigma(x), x \in W_{n}\right\}$. 
Пусть $\mu_{n}, n \geqslant 1,-$ последовательность мер на множествах $\Omega_{V_{n}}$, обладающая свойством согласованности (3). Тогда в силу теоремы Колмогорова существует, и притом единственная, предельная мера $\mu$ на $\Omega_{V}=\Omega$ (которая называется предельной мерой Гиббса) такая, что $\mu\left(\sigma_{n}\right)=\mu_{n}\left(\sigma_{n}\right)$ для каждого $n=1,2, \ldots$. Известно, что меры (2) удовлетворяют условию (3) тогда и только тогда, когда совокупность величин $h=\left\{h_{x}, x \in G_{k}\right\}$ удовлетворяет условию

$$
h_{x}=\lambda \beta+\sum_{y \in S(x)} f\left(h_{y}, \theta\right)
$$

где $S(x)$ - множество прямых потомков точки $x \in V$ (см. монографию [1]). Здесь

$$
f(x, \theta)=\operatorname{arcth}(\theta \operatorname{th} x), \quad \theta=\operatorname{th}(J \beta) .
$$

Пусть $G_{k} / \widehat{G}_{k}=\left\{H_{1}, \ldots, H_{r}\right\}-$ фактор-группа, где $\widehat{G}_{k}-$ нормальный делитель индекса $r \geqslant 1$.

ОПрЕДЕЛЕНиЕ 1. Совокупность величин $h=\left\{h_{x}, x \in G_{k}\right\}$ называется $\widehat{G}_{k}$-nерuодической, если $h_{x y}=h_{x}$ для любых $x \in G_{k}, y \in \widehat{G}_{k} . G_{k}$-периодическая мера называется трансляционно-инвариантной.

Для $x \in G_{k}$ введем обозначение $x_{\downarrow}=\left\{y \in G_{k} \mid\langle x, y\rangle\right\} \backslash S(x)$.

ОПрЕДЕЛЕНиЕ 2. Совокупность величин $h=\left\{h_{x}, x \in G_{k}\right\}$ назовем $\widehat{G}_{k}$-слабо периодической, если $h_{x}=h_{i j}$, при $x \in H_{i}, x_{\downarrow} \in H_{j}$ для любого $x \in G_{k}$.

Заметим, что слабо периодическая совокупность $h$ совпадает с обычной периодической (см. определение 1 ), если значение $h_{x}$ не зависит от $x_{\downarrow}$.

ОПРЕДЕЛЕНИЕ 3. Меру $\mu$ назовем $\widehat{G}_{k}$-(слабо) периодической, если она соответствует $\widehat{G}_{k}$-(слабо) периодической совокупности величин $h$.

В настоящей работе мы изучаем слабо периодические меры Гиббса и проверяем существование таких мер для модели Изинга с внешним полем.

\section{3. СЛАБО ПЕРИОДИЧЕСКИЕ МЕРЫ}

Трудность задачи описания слабо периодических мер Гиббса зависит от структуры и индекса нормального делителя, относительно которого требуется периодичность. В работе [12] доказано, что в группе $G_{k}$ не существует нормального делителя нечетного (отличного от единицы) индекса. Поэтому мы рассмотрим нормальные делители четного индекса. В настоящей работе мы ограничимся случаем индекса 2.

Опишем $\bar{G}_{k}$-слабо периодические меры Гиббса для любого нормального делителя $\bar{G}_{k}$ индекса 2. Заметим, что любой нормальный делитель индекса 2 группы $G_{k}$ имеет вид

$$
H_{A}=\left\{x \in G_{k} \mid \sum_{i \in A} w_{x}\left(a_{i}\right) \text { - четное число }\right\},
$$

где $\varnothing \neq A \subseteq N_{k}=\{1,2, \ldots, k+1\}$ и $w_{x}\left(a_{i}\right)$ - число букв $a_{i}$ в слове $x \in G_{k}[1]$. 
Пусть множество $A \subset\{1,2, \ldots, k+1\}$ и $H_{A}$ - соответствующий ему нормальный делитель индекса 2. Заметим, что в случае $|A|=k+1$ (где $|A|$ обозначает число элементов множества $A$ ), т. е. в случае $A=N_{k}$, понятие слабой периодичности совпадает с обычной периодичностью. Поэтому рассмотрим множество $A \subset N_{k}$ такое, что $A \neq N_{k}$. Тогда в силу (4) $G_{k}$-слабо периодическая совокупность $h$ имеет вид

$$
h_{x}=\left\{\begin{array}{lll}
h_{1}, & x \in H_{A}, & x_{\downarrow} \in H_{A}, \\
h_{2}, & x \in H_{A}, & x_{\downarrow} \in G_{k} \backslash H_{A}, \\
h_{3}, & x \in G_{k} \backslash H_{A}, & x_{\downarrow} \in H_{A}, \\
h_{4}, & x \in G_{k} \backslash H_{A}, & x_{\downarrow} \in G_{k} \backslash H_{A},
\end{array}\right.
$$

где $h_{i}, i=\overline{1,4}$, удовлетворяют системе уравнений

$$
\begin{aligned}
& h_{1}=\lambda \beta+|A| f\left(h_{3}, \theta\right)+(k-|A|) f\left(h_{1}, \theta\right), \\
& h_{2}=\lambda \beta+(|A|-1) f\left(h_{3}, \theta\right)+(k+1-|A|) f\left(h_{1}, \theta\right), \\
& h_{3}=\lambda \beta+(|A|-1) f\left(h_{2}, \theta\right)+(k+1-|A|) f\left(h_{4}, \theta\right), \\
& h_{4}=\lambda \beta+|A| f\left(h_{2}, \theta\right)+(k-|A|) f\left(h_{4}, \theta\right) .
\end{aligned}
$$

Рассмотрим отображение $W: \mathbb{R}^{4} \rightarrow \mathbb{R}^{4}$, заданное системой (6) так, что (6) есть уравнение $h=W(h)$. Отображение $W$ имеет инвариантные множества

$$
I_{1}=\left\{h \in \mathbb{R}^{4}: h_{1}=h_{2}=h_{3}=h_{4}\right\}, \quad I_{2}=\left\{h \in \mathbb{R}^{4}: h_{1}=h_{4}, h_{2}=h_{3}\right\} .
$$

Теорема 1. Имеют место следующие утверждения:

1) для модели Изинга с внешними полями все $H_{A}$-слабо периодические мерь Гиббса на множествах $I_{1}, I_{2}$ являются трансляиионно-инвариантными;

2) при $|A|=k u \theta>0$ все $H_{A}$-слабо периодические меры Гиббса являются трансляционно-инвариантными.

ДокАЗАтЕЛьство. 1. Достаточно показать, что система уравнений (6) имеет решение только вида $h_{1}=h_{2}=h_{3}=h_{4}$. Для инвариантного множества $I_{1}$ доказательство теоремы очевидно. Докажем теорему для инвариантного множества $I_{2}$.

Используя формулу

$$
f(h, \theta)=\operatorname{arcth}(\theta \operatorname{th} h)=\frac{1}{2} \ln \frac{(1+\theta) e^{2 h}+(1-\theta)}{(1-\theta) e^{2 h}+(1+\theta)}
$$

и вводя обозначения $\alpha=(1-\theta) /(1+\theta)$ и $z_{i}=e^{2 h_{i}}, i=\overline{1,4}$, вместо (6) получим следующую систему уравнений:

$$
\begin{aligned}
& z_{1}=e^{2 \lambda \beta}\left(\frac{z_{3}+\alpha}{\alpha z_{3}+1}\right)^{|A|}\left(\frac{z_{1}+\alpha}{\alpha z_{1}+1}\right)^{(k-|A|)}, \\
& z_{2}=e^{2 \lambda \beta}\left(\frac{z_{3}+\alpha}{\alpha z_{3}+1}\right)^{|A|-1}\left(\frac{z_{1}+\alpha}{\alpha z_{1}+1}\right)^{(k+1-|A|)}, \\
& z_{3}=e^{2 \lambda \beta}\left(\frac{z_{2}+\alpha}{\alpha z_{2}+1}\right)^{|A|-1}\left(\frac{z_{4}+\alpha}{\alpha z_{4}+1}\right)^{(k+1-|A|)}, \\
& z_{4}=e^{2 \lambda \beta}\left(\frac{z_{2}+\alpha}{\alpha z_{2}+1}\right)^{|A|}\left(\frac{z_{4}+\alpha}{\alpha z_{4}+1}\right)^{(k-|A|)} .
\end{aligned}
$$


Нетрудный, но достаточно громоздкий анализ показывает, что эту систему можно привести к виду

$$
\begin{aligned}
& z_{1}-z_{2}=A_{1}\left(z_{3}-z_{1}\right) \\
& z_{1}-z_{3}=A_{2}\left(z_{1}-z_{4}\right)+B_{2}\left(z_{3}-z_{4}\right)+C_{2}\left(z_{3}-z_{2}\right), \\
& z_{1}-z_{4}=A_{3}\left(z_{1}-z_{4}\right)+B_{3}\left(z_{3}-z_{2}\right) \\
& z_{2}-z_{3}=A_{4}\left(z_{3}-z_{2}\right)+B_{4}\left(z_{1}-z_{4}\right) \\
& z_{2}-z_{4}=A_{5}\left(z_{3}-z_{2}\right)+B_{5}\left(z_{1}-z_{2}\right)+C_{5}\left(z_{1}-z_{4}\right), \\
& z_{3}-z_{4}=A_{6}\left(z_{4}-z_{2}\right)
\end{aligned}
$$

где

$$
\begin{aligned}
A_{i} & =\left(1-\alpha^{2}\right) \widetilde{A}_{i}\left(z_{1}, z_{2}, z_{3}, z_{4}\right), \\
B_{i} & =\left(1-\alpha^{2}\right) \widetilde{B}_{i}\left(z_{1}, z_{2}, z_{3}, z_{4}\right), \\
C_{i} & =\left(1-\alpha^{2}\right) \widetilde{C}_{i}\left(z_{1}, z_{2}, z_{3}, z_{4}\right)
\end{aligned}
$$

и $\widetilde{A}_{i}, \widetilde{B}_{i}, \widetilde{C}_{i}$ положительны для всех $i=\overline{1,6}$.

На инвариантном множестве $I_{2}$ мы имеем $h_{2}=h_{3}$, откуда при $\alpha<1$ из равенства $z_{1}-z_{2}=A_{1}\left(z_{3}-z_{1}\right)$ следует, что $z_{1}=z_{2}$.

В антиферромагнитном случае, т. е. при $\alpha \in(1,+\infty)$ получаем $A_{i}, B_{i}, C_{i}<0$ для всех $i$. Тогда на инвариантном множестве $I_{2}$ выполняется $h_{2}=h_{3}$, т. е. $z_{2}=z_{3}$, отсюда с учетом $z_{2}-z_{4}=A_{3}\left(z_{1}-z_{4}\right)$ следует, что $z_{1}=z_{4}$. Следовательно, при всех $\alpha \in(0,+\infty)$ имеем $z_{1}=z_{2}$, откуда $z_{1}=z_{2}=z_{3}=z_{4}$ на множестве $I_{2}$.

2. В случае $|A|=k$ из (6) получим

$$
\begin{aligned}
& h_{2}=\lambda \beta+(k-1) f\left(h_{3}, \theta\right)+f\left(\lambda \beta+k f\left(h_{3}, \theta\right), \theta\right), \\
& h_{3}=\lambda \beta+(k-1) f\left(h_{2}, \theta\right)+f\left(\lambda \beta+k f\left(h_{2}, \theta\right), \theta\right) .
\end{aligned}
$$

Теперь докажем, что эта система имеет только решения вида $h_{2}=h_{3}$. Пусть $h_{2}>h_{3}$, тогда из (10) имеем

$$
h_{2}-h_{3}=(k-1)\left(f\left(h_{3}, \theta\right)-f\left(h_{2}, \theta\right)\right)+f\left(\lambda \beta+k f\left(h_{3}, \theta\right), \theta\right)-f\left(\lambda \beta+k f\left(h_{2}, \theta\right), \theta\right) .
$$

Легко проверить, что функция $f$ при $\theta>0$ строго возрастает. Следовательно, равенство (11) не может быть верным, так как его левая часть положительная, а правая отрицательная. В случае $h_{2}<h_{3}$ равенство (11) также не выполняется, следовательно, $h_{2}=h_{3}$, что дает трансляционно-инвариантные решения системы (6). Теорема доказана.

Теперь рассмотрим антиферромагнитную модель Изинга с внешним полем, т. е. случай $\alpha>1(\theta<0)$. Введем следующие обозначения:

$$
a=e^{2 \lambda \beta}, \quad \varphi(x)=\frac{x+\alpha}{\alpha x+1} .
$$

Известно [1], [6], [7], что в этом случае существует единственная трансляционно-инвариатная мера Гиббса, которая соответствует единственному решению уравнения

$$
z=a \varphi^{k}(z)
$$

это решение обозначим через $z_{*}$. 
Предположим, что $|A|=k$, тогда система уравнений (8) записывается как

$$
\begin{aligned}
z_{1} & =a \varphi^{k}\left(z_{3}\right), & z_{2} & =a \varphi^{k-1}\left(z_{3}\right) \varphi\left(z_{1}\right), \\
z_{3} & =a \varphi^{k-1}\left(z_{2}\right) \varphi\left(z_{4}\right), & z_{4} & =a \varphi^{k}\left(z_{2}\right) .
\end{aligned}
$$

Решение системы уравнений (12) сводится к анализу следующей системы уравнений:

$$
\begin{aligned}
& z_{2}=a \varphi^{k-1}\left(z_{3}\right) \varphi\left(a\left(\varphi^{k}\left(z_{3}\right)\right)\right), \\
& z_{3}=a \varphi^{k-1}\left(z_{2}\right) \varphi\left(a\left(\varphi^{k}\left(z_{2}\right)\right)\right) .
\end{aligned}
$$

Если ввести обозначение

$$
\psi(z)=a \varphi^{k-1}(z) \varphi\left(a\left(\varphi^{k}(z)\right)\right)
$$

то система уравнений (13) приобретает вид

$$
z_{2}=\psi\left(z_{3}\right), \quad z_{3}=\psi\left(z_{2}\right)
$$

Эта система имеет столько решений, сколько имеет решений уравнение $\psi(\psi(z))=z$.

Лемма 1. Пусть $\gamma:[0,1] \rightarrow[0,1]$ есть непрерывная функиия с неподвижной точкой $\xi \in(0,1)$. Предположим, что функция $\gamma$ дифференцируема в точке $\xi$ и $\gamma^{\prime}(\xi)<-1$. Тогда существуют значения $x_{0}, x_{1}$ такие, что выполнены неравенства $0 \leqslant x_{0}<\xi<x_{1} \leqslant 1$ и $\gamma\left(x_{0}\right)=x_{1}, \gamma\left(x_{1}\right)=x_{0}$.

ДоКАЗАТЕЛЬСТво этой леммы можно найти в монографии [13].

Легко видеть, что для функции (14) верны следующие утверждения: она определена на $\mathbb{R}_{+}$, является ограниченной, дифференцируемой и $\psi\left(z_{*}\right)=z_{*}$. Тогда по лемме 1 при $\psi^{\prime}\left(z_{*}\right)<-1$ система уравнений (15) имеет три решения вида $\left(z_{*}, z_{*}\right)$, $\left(z_{0}, z_{1}\right),\left(z_{1}, z_{0}\right)$, где $\psi\left(z_{0}\right)=z_{1}, \psi\left(z_{1}\right)=z_{0}$. Неравенство $\psi^{\prime}\left(z_{*}\right)<-1$ эквивалентно следующему неравенству:

$$
k \frac{\left(1-\alpha^{2}\right)^{2} z_{*}^{2(k-1) / k}}{\left(\alpha z_{*}+1\right)^{4}}+b(k-1) \frac{\left(1-\alpha^{2}\right) z_{*}^{(k-1) / k}}{\left(\alpha z_{*}+1\right)^{2}}+b^{2}<0,
$$

где $b=\sqrt[k]{a}$. Следовательно, $\left(b-b_{1}\right)\left(b-b_{2}\right)<0$, где

$$
\begin{aligned}
& b_{1}=\frac{\left(k-1-\sqrt{k^{2}-6 k+1}\right)\left(\alpha^{2}-1\right) z_{*}^{(k-1) / k}}{2\left(\alpha z_{*}+1\right)^{2}}, \\
& b_{2}=\frac{\left(k-1+\sqrt{k^{2}-6 k+1}\right)\left(\alpha^{2}-1\right) z_{*}^{(k-1) / k}}{2\left(\alpha z_{*}+1\right)^{2}} .
\end{aligned}
$$

В результате доказана следующая

Теорема 2. При $|A| \geqslant 6 u \lambda \in\left(\lambda_{1}, \lambda_{2}\right)$, где $\lambda_{1,2}=(k / 2 \beta) \ln b_{1,2}$ и величины $b_{1,2}$ определены в (17), для антиферромагнитной модели Изинга с внешним полем существует не менее двух $H_{A}$-слабо периодических (непериодических) мер Гиббса. 
Для модели Изинга в работе [14] доказано существование не менее чем двух слабо периодических (непериодических) мер Гиббса, и теорема 2 обобщает этот результат на случай модели Изинга с внешним полем. Действительно, если рассмотреть модель Изинга с нулевым внешним полем, т. е. при $a=1$, неравенство (16) принимает следующий вид:

$$
k \frac{(1-\alpha)^{2}}{(1+\alpha)^{2}}+(k+1) \frac{(1-\alpha)}{(1+\alpha)}+1<0 .
$$

Отсюда получим $\alpha \in\left(\alpha_{1}, \alpha_{2}\right)$, где $\alpha_{1,2}=\left(k-1 \pm \sqrt{k^{2}-6 k+1}\right) / 2$, т. е. мы имеем результат работы [14].

ЗАмЕчАниЕ 1 . Полученные $H_{A}$-слабо периодические меры в теореме 2 являются новыми и дают возможность описать континуум непериодических мер Гиббса, отличных от ранее известных.

Благодарности. Автор выражает свою глубокую признательность профессору У. А. Розикову за постановку задачи и полезные советы по работе.

\section{Список литературы}

[1] U. A. Rozikov, Gibbs Measures on Cayley Trees, World Sci., Singapore, 2013.

[2] П. М. Блехер, Н. Н. Ганиходжаев, ТВП, 35:2 (1990), 220-230.

[3] F. Spitzer, Ann. Probab., 3 (1975), 387-398.

[4] S. Zachary, Ann. Probab., 11:4 (1983), 894-903.

[5] У. А. Розиков, ТМФ, 118:1 (1999), 95-104.

[6] Х.-О. Георги, Гиббсовские меры и фазовые переходы, Мир, М., 1992.

[7] C. Preston, Gibbs States on Countable Sets, Cambridge Univ. Press, London, 1974.

[8] У. А. Розиков, М. М. Рахматуллаев, ТМФ, 156:2 (2008), 292-302.

[9] У. А. Розиков, М. М. Рахматуллаев, ТМФ, 160:3 (2009), 507-516.

[10] У. А. Розиков, М. М. Рахматуллаев, Докл. АН РУз, 4 (2008), 12-15.

[11] М. М. Рахматуллаев, Узб. матем. журн., 2 (2009), 144-152.

[12] Э. П. Норматов, У. А. Розиков, Матем. заметки, 79:3 (2006), 434-443.

[13] H. Kesten, Adv. Appl. Probab., 2 (1970), 1-82.

[14] M. Rahmatullaev, On new weakly periodic Gibbs measures of Ising model on a Cayley tree, arXiv: 1409.4553 . 